\title{
Orthogonal polynomials on the unit circle: Verblunsky coefficients with some restrictions imposed on a pair of related real sequences
}

\author{
Cleonice F. Bracciali ${ }^{1}$ - Jairo S. Silva ${ }^{2}$. \\ A. Sri Ranga ${ }^{1}$. Daniel O. Veronese ${ }^{3}$
}

Received: 24 August 2016 / Accepted: 12 October 2016 / Published online: 22 October 2016

(C) SBMAC - Sociedade Brasileira de Matemática Aplicada e Computacional 2016

\begin{abstract}
It was shown recently that associated with a pair of real sequences $\left\{\left\{c_{n}\right\}_{n=1}^{\infty}\right.$, $\left.\left\{d_{n}\right\}_{n=1}^{\infty}\right\}$, with $\left\{d_{n}\right\}_{n=1}^{\infty}$ a positive chain sequence, there exists a unique nontrivial probability measure $\mu$ on the unit circle. The Verblunsky coefficients $\left\{\alpha_{n}\right\}_{n=0}^{\infty}$ associated with the orthogonal polynomials with respect to $\mu$ are given by the relation

$$
\alpha_{n-1}=\bar{\tau}_{n-1}\left[\frac{1-2 m_{n}-i c_{n}}{1-i c_{n}}\right], \quad n \geq 1,
$$

where $\tau_{0}=1, \tau_{n}=\prod_{k=1}^{n}\left(1-i c_{k}\right) /\left(1+i c_{k}\right), n \geq 1$ and $\left\{m_{n}\right\}_{n=0}^{\infty}$ is the minimal parameter sequence of $\left\{d_{n}\right\}_{n=1}^{\infty}$. In this manuscript, we consider this relation and its consequences by imposing some restrictions of sign and periodicity on the sequences $\left\{c_{n}\right\}_{n=1}^{\infty}$ and $\left\{m_{n}\right\}_{n=1}^{\infty}$. When the sequence $\left\{c_{n}\right\}_{n=1}^{\infty}$ is of alternating sign, we use information about the zeros of associated para-orthogonal polynomials to show that there is a gap in the support of the
\end{abstract}

Communicated by Antonio José Silva Neto.

C. F. Bracciali and A. S. Ranga were supported by funds from FAPESP (2014/22571-2) and CNPq (475502/2013-2, 305073/2014-1, 305208/2015-2) of Brazil. J. S. Silva and D. O. Veronese were supported by grants from CAPES of Brazil.

$凶 \quad$ Jairo S. Silva

jairo.santos@ufma.br

Cleonice F. Bracciali

cleonice@ibilce.unesp.br

\section{A. Sri Ranga}

ranga@ibilce.unesp.br

Daniel O. Veronese

veronese@icte.uftm.edu.br

1 Departamento de Matemática Aplicada, IBILCE, UNESP - Universidade Estadual Paulista, São José do Rio Preto, SP 15054-000, Brazil

2 Departamento de Matemática, Universidade Federal do Maranhão, São Luís, MA 65080-805, Brazil

3 ICTE, Universidade Federal do Triângulo Mineiro, Uberaba, MG 38064-200, Brazil

国 springer $\mathscr{D} / \mathfrak{A} \mathbb{A}$ 
measure in the neighbourhood of $z=-1$. Furthermore, we show that it is possible to generate periodic Verblunsky coefficients by choosing periodic sequences $\left\{c_{n}\right\}_{n=1}^{\infty}$ and $\left\{m_{n}\right\}_{n=1}^{\infty}$ with the additional restriction $c_{2 n}=-c_{2 n-1}, n \geq 1$. We also give some results on periodic Verblunsky coefficients from the point of view of positive chain sequences. An example is provided to illustrate the results obtained.

Keywords Para-orthogonal polynomials · Probability measures · Periodic Verblunsky coefficients · Chain sequences $\cdot$ Alternating sign sequences

Mathematics Subject Classification $42 \mathrm{C} 05 \cdot 33 \mathrm{C} 47$

\section{Introduction}

Orthogonal polynomials on the unit circle (OPUC, in short) have been commonly known as Szegő polynomials in honor of Gábor Szegő who introduced them in the first half of the twentieth century. Because of their applications in quadrature rules, signal processing, operator and spectral theory and many other topics, these polynomials have received a lot of attention in recent years (see, for example, Breuer et al. 2010; Castillo et al. 2011; Costa et al. 2011; Kheifets et al. 2011; Peherstorfer 2011; Peherstorfer et al. 2009; Simanek 2012; Tsujimoto and Zhedanov 2009). For many years, a firsthand text for an introduction to these polynomials has been the classical book (Szegó 1975) of Szegó. However, for recent and more up-to-date texts on this subject, we refer to the two volumes of Simon (2005a, b). For further interesting reading on this subject, we refer to Chapter 8 of Ismail's recent book (Ismail 2005).

Given a nontrivial probability measure $\mu(z)=\mu\left(e^{i \theta}\right)$ on the unit circle $\mathbb{T}=\left\{z=e^{i \theta}\right.$ : $0 \leq \theta \leq 2 \pi\}$, the associated sequences of OPUC $\left\{\phi_{n}\right\}$ are those with the property

$$
\int_{\mathbb{T}} \bar{z}^{j} \phi_{n}(z) d \mu(z)=\int_{0}^{2 \pi} e^{-i j \theta} \phi_{n}\left(e^{i \theta}\right) d \mu\left(e^{i \theta}\right)=0, \quad 0 \leq j \leq n-1, \quad n \geq 1 .
$$

Letting $\kappa_{n}^{-2}=\left\|\phi_{n}\right\|^{2}=\int_{\mathbb{T}}\left|\phi_{n}(z)\right|^{2} d \mu(z)$, the orthonormal polynomials on the unit circle are $\varphi_{n}(z)=\kappa_{n} \phi_{n}(z), n \geq 0$.

The polynomials $\phi_{n}(z), n \geq 0$, considered as monic polynomials, satisfy the so-called forward and backward recurrence relations, respectively,

$$
\begin{aligned}
& \phi_{n}(z)=z \phi_{n-1}(z)-\bar{\alpha}_{n-1} \phi_{n-1}^{*}(z), \\
& \phi_{n}(z)=\left(1-\left|\alpha_{n-1}\right|^{2}\right) z \phi_{n-1}(z)-\bar{\alpha}_{n-1} \phi_{n}^{*}(z), \quad n \geq 1,
\end{aligned}
$$

where $\alpha_{n-1}=-\overline{\phi_{n}(0)}$ and $\phi_{n}^{*}(z)=z^{n} \overline{\phi_{n}(1 / \bar{z})}$ denotes the reversed (reciprocal) polynomial of $\phi_{n}(z)$. The numbers $\alpha_{n}$, in recent years, have been referred to as Verblunsky coefficients. It is known that these coefficients are such that $\left|\alpha_{n}\right|<1, n \geq 0$. Moreover, the OPUC and the associated measure are completely determined from these coefficients (see for example Simon 2005a, Theorem 1.7.11). A very nice and short constructive proof of this last statement can be found in Erdélyi et al. (1991).

Another entity that has recently played an important role in the theory of OPUC are the positive chain sequences. According to Chihara (1978), a sequence $\left\{a_{n}\right\}_{n=1}^{\infty}$ is a positive chain sequence if there exists a second sequence $\left\{g_{n}\right\}_{n=0}^{\infty}$ such that

$$
0 \leq g_{0}<1, \quad 0<g_{n}<1, \text { for } n \geq 1, \quad \text { and } a_{n}=\left(1-g_{n-1}\right) g_{n} \text {, for } n \geq 1 \text {. }
$$


The sequence $\left\{g_{n}\right\}_{n=0}^{\infty}$ (which can be non-unique) is called a parameter sequence of the positive chain sequence $\left\{a_{n}\right\}_{n=1}^{\infty}$. It is known (see, for example, Chihara 1978) that every positive chain sequence has a minimal parameter sequence denoted by $\left\{m_{n}\right\}_{n=0}^{\infty}$, obtained when $m_{0}=0$. Moreover, every positive chain sequence has a maximal parameter sequence denoted by $\left\{M_{n}\right\}_{n=0}^{\infty}$, which is characterized by the condition that if $g_{0}>M_{0}$, then $\left\{g_{n}\right\}_{n=1}^{\infty}$ generated by $g_{n}=a_{n} /\left(1-g_{n-1}\right), n \geq 1$ does not satisfy $0<g_{n}<1, n \geq 1$.

It was shown in Costa et al. (2013) that given any nontrivial probability measure on the unit circle, then corresponding to this measure there exists a pair of real sequences $\left\{c_{n}\right\}_{n=1}^{\infty}$ and $\left\{d_{n}\right\}_{n=1}^{\infty}$, where $\left\{d_{n}\right\}_{n=1}^{\infty}$ is also a positive chain sequence. In Theorem 1, we have given complete information regarding this statement and its reciprocal. To be precise, the sequences $\left\{c_{n}\right\}_{n=1}^{\infty}$ and $\left\{d_{n}\right\}_{n=1}^{\infty}$ are the coefficients of the three term recurrence relation

$$
R_{n+1}(z)=\left[\left(1+i c_{n+1}\right) z+\left(1-i c_{n+1}\right)\right] R_{n}(z)-4 d_{n+1} z R_{n-1}(z), \quad n \geq 1,
$$

with $R_{0}(z)=1$ and $R_{1}(z)=\left(1+i c_{1}\right) z+\left(1-i c_{1}\right)$, where

$$
R_{n}(z)=\frac{\prod_{j=1}^{n}\left[1-\tau_{j-1} \alpha_{j-1}\right]}{\prod_{j=1}^{n}\left[1-\mathcal{R} e\left(\tau_{j-1} \alpha_{j-1}\right)\right]} \frac{z \phi_{n}(z)-\tau_{n} \phi_{n}^{*}(z)}{z-1},
$$

with $\tau_{n}=\phi_{n}(1) / \phi_{n}^{*}(1), n \geq 0$.

From the sequences $\left\{c_{n}\right\}_{n=1}^{\infty}$ and $\left\{d_{n}\right\}_{n=1}^{\infty}$, it is possible to recover the associated probability measure using certain rational functions that follow from the recurrence formula (1). In Castillo et al. (2014), using standard arguments involving continued fractions, series expansions at infinity and at the origin, and Helly's Selection Theorem, the associated measure $\mu$ is given as a limit of a subsequence of discrete measures $\psi_{n}\left(e^{i \theta}\right)$ whose pure points (those different from $z=1$ ) are exactly the zeros of $R_{n}(z)$. The results given in Castillo et al. (2014) enable us to give information about the support of the measure $\mu$ by analysing the zeros $z_{n, j}=e^{i \theta_{n, j}}, j=1,2 \ldots, n$, of $R_{n}(z)$, or, equivalently, by analysing the zeros of the functions $\mathcal{W}_{n}(x)$, given by

$$
\mathcal{W}_{n}(x)=2^{-n} e^{-i n \theta / 2} R_{n}\left(e^{i \theta}\right), \quad n \geq 0,
$$

where $x=\cos (\theta / 2)$. The sequence of functions $\left\{\mathcal{W}_{n}\right\}_{n=0}^{\infty}$ satisfies the three term recurrence relation (see Bracciali et al. 2016; Dimitrov and Sri Ranga 2013)

$$
\mathcal{W}_{n+1}(x)=\left(x-c_{n+1} \sqrt{1-x^{2}}\right) \mathcal{W}_{n}(x)-d_{n+1} \mathcal{W}_{n-1}(x), \quad n \geq 1,
$$

with $\mathcal{W}_{0}(x)=1$ and $\mathcal{W}_{1}(x)=x-c_{1} \sqrt{1-x^{2}}$.

For any $n \geq 1, \mathcal{W}_{n}(x)$ has exactly $n$ distinct zeros $x_{n, j}=\cos \left(\theta_{n, j} / 2\right), j=1,2 \ldots, n$, in $(-1,1)$. We mention that the proof given in Dimitrov and Sri Ranga (2013) for the interlacing property of the zeros of $R_{n}(z)$ and $R_{n+1}(z)$ is by proving the interlacing property

$$
-1<x_{n+1, n+1}<x_{n, n}<x_{n+1, n}<\cdots<x_{n, 1}<x_{n+1,1}<1, \quad n \geq 1,
$$

for the zeros of $\mathcal{W}_{n}(x)$ and $\mathcal{W}_{n+1}(x)$ using the three term recurrence relation (3).

The aim of this manuscript is to study the sequences of Verblunsky coefficients where the related sequences $\left\{c_{n}\right\}_{n=1}^{\infty}$ and $\left\{m_{n}\right\}_{n=1}^{\infty}$ have restrictions of sign and periodicity. We show that, under certain conditions, it is possible to estimate the support of the associated measure and to get periodic Verblunsky coefficients. Furthermore, we discuss some geometric aspects related to these restrictions.

This manuscript is organized as follows. In Sect. 2, we give a summary of all the required theoretical results. Section 3 deals with the results concerning the measures for which the 
associated sequence $\left\{c_{n}\right\}_{n=1}^{\infty}$ has the alternating sign property, namely, $c_{n}=(-1)^{n} \tilde{c}_{n}$, for $n \geq 1$, where $\tilde{c}_{n}$ is a positive (or negative) sequence of real numbers. In Sect. 4 , some results regarding the measures associated with the sequence of periodic Verblunsky coefficients are established. Finally, in Sect. 5, we give an example to illustrate the results obtained.

\section{Some preliminary results}

In this section, we present some results concerning nontrivial probability measures and positive chain sequences [for more details on chain sequences, we refer to Chihara (1978) and Wall (1948)]. Furthermore, some known results about measures with associated periodic Verblunsky coefficients are presented.

We begin with two theorems established in Costa et al. (2013). The first theorem provides a characterization for nontrivial probability measures in terms of two sequences, $\left\{c_{n}\right\}_{n=1}^{\infty}$ and $\left\{d_{n}\right\}_{n=1}^{\infty}$.

\section{Theorem 1 (Costa et al. 2013)}

(a) Given a nontrivial probability measure $\mu$ on the unit circle, then associated with it there exists a unique pair of real sequences $\left\{\left\{c_{n}\right\}_{n=1}^{\infty},\left\{d_{n}\right\}_{n=1}^{\infty}\right\}$, where $\left\{d_{n}\right\}_{n=1}^{\infty}$ is also a positive chain sequence. Specifically, if $\left\{\alpha_{n}\right\}_{n=0}^{\infty}$ is the associated sequence of Verblunsky coefficients and if the sequence $\tau_{n}$ is such that

$$
\tau_{0}=1 \text { and } \tau_{n}=\tau_{n-1} \frac{1-\bar{\tau}_{n-1} \bar{\alpha}_{n-1}}{1-\tau_{n-1} \alpha_{n-1}}, \quad n \geq 1,
$$

then $m_{0}=0$,

$$
c_{n}=\frac{-\operatorname{Im}\left(\tau_{n-1} \alpha_{n-1}\right)}{1-\operatorname{Re}\left(\tau_{n-1} \alpha_{n-1}\right)} \quad \text { and } m_{n}=\frac{1}{2} \frac{\left|1-\tau_{n-1} \alpha_{n-1}\right|^{2}}{\left[1-\operatorname{Re}\left(\tau_{n-1} \alpha_{n-1}\right)\right]}, \quad n \geq 1,
$$

where $\left\{m_{n}\right\}_{n=0}^{\infty}$ is the minimal parameter sequence of $\left\{d_{n}\right\}_{n=1}^{\infty}$. Moreover, the maximal parameter sequence $\left\{M_{n}\right\}_{n=0}^{\infty}$ of $\left\{d_{n}\right\}_{n=1}^{\infty}$ is such that $M_{0}$ is the value of the jump in the measure at $z=1$.

(b) Conversely, given a pair of real sequences $\left\{\left\{c_{n}\right\}_{n=1}^{\infty},\left\{d_{n}\right\}_{n=1}^{\infty}\right\}$, where $\left\{d_{n}\right\}_{n=1}^{\infty}$ is also a positive chain sequence, then associated with this pair there exists a unique nontrivial probability measure $\mu$ supported on the unit circle. Specifically, if $\left\{m_{n}\right\}_{n=0}^{\infty}$ is the minimal parameter sequence of $\left\{d_{n}\right\}_{n=1}^{\infty}$, then $\tau_{0}=1$,

$$
\tau_{n-1} \alpha_{n-1}=\frac{1-2 m_{n}-i c_{n}}{1-i c_{n}} \text { and } \tau_{n}=\frac{1-i c_{n}}{1+i c_{n}} \tau_{n-1}, \quad n \geq 1 .
$$

Moreover, the measure has a jump $M_{0}$ at $z=1$, where $\left\{M_{n}\right\}_{n=0}^{\infty}$ is the maximal parameter sequence of $\left\{d_{n}\right\}_{n=1}^{\infty}$.

The next theorem gives information regarding the pure points. This theorem is obtained as a consequence of Wall's criterion for maximal parameter sequence of positive chain sequences.

Theorem 2 (Costa et al. 2013) The probability measure $\mu$ has a pure point at $w(|w|=1)$ if, and only if,

$$
\sum_{n=1}^{\infty}\left[\prod_{j=1}^{n} \frac{\left|1-w \tau_{j-1}(w) \alpha_{j-1}\right|^{2}}{1-\left|\alpha_{j-1}\right|^{2}}\right]=\lambda(w)<\infty
$$


Moreover, the size of the mass at the point $z=w$ is equal to $t=[1+\lambda(w)]^{-1}$. Here, $\tau_{0}(w)=1$ and

$$
\tau_{j+1}(w)=\frac{\phi_{j+1}(w)}{\phi_{j+1}^{*}(w)}=\frac{w \tau_{j}(w)-\bar{\alpha}_{j}}{1-w \tau_{j}(w) \alpha_{j}}, \quad j \geq 0 .
$$

Now, we discuss a result obtained in Castillo et al. (2014), which leads to a relation between the zeros of the polynomials $R_{n}(z)$ and the measure associated with the pair of real sequences $\left\{\left\{c_{n}\right\}_{n=1}^{\infty},\left\{d_{n}\right\}_{n=1}^{\infty}\right\}$.

Consider the new sequence of polynomials $\left\{Q_{n}\right\}$ satisfying

$$
Q_{n+1}(z)=\left[\left(1+i c_{n+1}\right) z+\left(1-i c_{n+1}\right)\right] Q_{n}(z)-4 d_{n+1} z Q_{n-1}(z), \quad n \geq 1,
$$

with $Q_{0}(z)=0$ and $Q_{1}(z)=2 d_{1}$.

Let $z_{n, j}=e^{i \theta_{n, j}}$ be the zeros of $R_{n}(z), \lambda_{n, 0}=1-\frac{Q_{n}(1)}{R_{n}(1)}$ and $\lambda_{n, j}=\frac{Q_{n}\left(z_{n, j}\right)}{\left(1-z_{n, j}\right) R_{n}^{\prime}\left(z_{n, j}\right)}$, with $j \in\{1,2, \ldots, n\}$. Thus, as shown in Castillo et al. (2014), $\sum_{j=0}^{n} \lambda_{n, j}=1$ and also $\lambda_{n, j}>0, j=0,1,2, \ldots, n$.

In addition, if we define the sequence of step-functions $\psi_{n}\left(e^{i \theta}\right), n \geq 1$, on $[0,2 \pi]$ by

$$
\psi_{n}\left(e^{i \theta}\right)= \begin{cases}0, & \theta=0, \\ \lambda_{n, 0}, & 0<\theta \leq \theta_{n, 1}, \\ \sum_{j=0}^{k} \lambda_{n, j}, & \theta_{n, k}<\theta \leq \theta_{n, k+1}, \quad k=1,2, \ldots, n-1, \\ 1, & \theta_{n, n}<\theta \leq 2 \pi\end{cases}
$$

then by Helly's Selection Theorem a subsequence of $\psi_{n}\left(e^{i \theta}\right)$ converges to the measure $\mu\left(e^{i \theta}\right)$ associated with the pair $\left\{\left\{c_{n}\right\}_{n=1}^{\infty},\left\{d_{n}\right\}_{n=1}^{\infty}\right\}$ as established in Theorem 1.

As an immediate consequence of this result, we can state the following.

Theorem 3 Let $\left\{\left\{c_{n}\right\}_{n=1}^{\infty},\left\{d_{n}\right\}_{n=1}^{\infty}\right\}$ be a pair of real sequences with $\left\{d_{n}\right\}_{n=1}^{\infty}$ a positive chain sequence. Moreover, let $R_{n}(z)$ be the sequence of polynomials given by (1) and let $\mu$ be the measure associated with this pair of sequences. In addition, suppose that the zeros of $R_{n}(z)$ lie on a closed arc $\mathcal{B}$ of the unit circle, for $n \geq 1$. Then, the support of the measure $\mu$ lies within $\mathcal{B} \cup\{1\}$.

We now present a review of basic results on measures with associated sequence of periodic Verblunsky coefficients. For more details regarding these results, we refer to Geronimus (1944), Peherstorfer and Steinbauer (1996a, b) and Simon (2005b).

Let $\left\{\alpha_{n}\right\}_{n=0}^{\infty}$ be a $p$-periodic sequence $\left(\alpha_{n+p}=\alpha_{n}, n \geq 0\right)$ of Verblunsky coefficients associated with the measure denoted by $\mu^{(p)}$ (here, $p$ is a fixed natural number). Consider the discriminant function $\Delta(z)=z^{-p / 2} \operatorname{Tr}\left(T_{p}(z)\right)$ where

$$
\begin{gathered}
T_{p}(z)=A\left(\alpha_{p-1}, z\right) \ldots A\left(\alpha_{0}, z\right), \\
A\left(\alpha_{j}, z\right)=\left(1-\left|\alpha_{j}\right|^{2}\right)^{-1 / 2}\left(\begin{array}{cc}
z & -\bar{\alpha}_{j} \\
-\alpha_{j} z & 1
\end{array}\right), \quad j=0, \ldots, p-1,
\end{gathered}
$$

and $\operatorname{Tr}\left(T_{p}(z)\right)$ denotes the trace of $T_{p}(z)$.

It is well known that all the $p$ distinct solutions of the equation $\Delta(z)=2$, which we denote by $z_{1}^{+}, \ldots, z_{p}^{+}$, lie on the unit circle $\mathbb{T}$. In the same way, the $p$ distinct solutions of the equation $\Delta(z)=-2$, denoted by $z_{1}^{-}, \ldots z_{p}^{-}$, also lie on $\mathbb{T}$. Using these solutions, it is possible to show that the unit circle can be decomposed into $2 p$ alternating sets $G_{1}, B_{1}, G_{2}, \ldots, B_{p}$ with each gap, $G_{j}$, open and each band, $B_{j}$, closed. Moreover, each band $B_{j}$ is given by $B_{j}=\left\{z \in \mathbb{T} \mid \arg \left(z_{j}^{\sigma_{j}}\right) \leq \arg (z) \leq \arg \left(z_{j}^{-\sigma_{j}}\right)\right\}$ with $\sigma_{j}=(-1)^{j+1}, j=1,2, \ldots, p$. 
Now, we mention four fundamental results (see Simon 2005b, Chapter 11) which give a completely characterization of probability measures on the unit circle associated with periodic Verblunsky coefficients. The first result provides information about the absolutely continuous part and the singular part of the measure.

Theorem 4 (Simon 2005b) Let $\left\{\alpha_{j}\right\}_{j=0}^{\infty}$ be a sequence of Verblunsky coefficients of period $p$ and let $d \mu^{(p)}=w(\theta) \frac{d \theta}{2 \pi}+d \mu_{s}^{(p)}$ be the associated probability measure. Then, if $B_{1}, \ldots, B_{p}$ are the corresponding bands we have that $\cup B_{j}$ is the essential support of the absolutely continuous spectrum and $d \mu_{s}^{(p)}\left[\cup B_{j}\right]=\emptyset$. Moreover, in each disjoint open arc on $\mathbb{T} \backslash \cup_{j=1}^{p}$ $B_{j}, \mu^{(p)}$ has either no support or a single pure point.

The next theorem provides information about the associated weight function $w(\theta)$.

Theorem 5 (Simon 2005b) Let $\left\{\alpha_{j}\right\}_{j=0}^{\infty}$ be a sequence of Verblunsky coefficients of period $p$ and let $d \mu^{(p)}=w(\theta) \frac{d \theta}{2 \pi}+d \mu_{s}^{(p)}$ be the associated measure. Then, for $e^{i \theta} \in \cup B_{j}$,

$$
w(\theta)=\frac{\sqrt{4-\Delta^{2}\left(e^{i \theta}\right)}}{2\left|\operatorname{Im}\left(e^{-i p \theta / 2}\right) \varphi_{p}\left(e^{i \theta}\right)\right|} .
$$

In particular,

(i) On $\cup B_{j}^{\text {int }}, w(\theta)>0$.

(ii) At an edge of a band that is by a closed gap (a gap which is empty), $w(\theta)>0$.

(iii) At an edge, $\theta_{0}$, of a band that is by an open gap, $w(\theta) \sim c\left(\theta-\theta_{0}\right)^{\frac{1}{2}}$ if $\varphi_{p}^{*}\left(e^{i \theta_{0}}\right)-$ $\varphi_{p}\left(e^{i \theta_{0}}\right) \neq 0$.

(iv) At an edge, $\theta_{0}$, of a band that is by an open gap, $w(\theta) \sim c\left(\theta-\theta_{0}\right)^{-\frac{1}{2}}$ if $\varphi_{p}^{*}\left(e^{i \theta_{0}}\right)-$ $\varphi_{p}\left(e^{i \theta_{0}}\right)=0$.

Finally, the following two theorems lead to a complete characterization for the pure points of the measure.

Theorem 6 (Simon 2005b) Let $\left\{\alpha_{j}\right\}_{j=0}^{\infty}$ be a sequence of Verblunsky coefficients of period p. Then,

$$
\pi(z)=\varphi_{p}^{*}(z)-\varphi_{p}(z)
$$

has all its zeros in the set of gap closures, one in each gap closure.

Theorem 7 (Simon 2005b) Let $\left\{\alpha_{j}\right\}_{j=0}^{\infty}$ be a sequence of Verblunsky coefficients of period $p$ and $\mu^{(p)}$ be the associated measure. Let $\theta_{0}$ be a point in a gap closure where $\varphi_{p}^{*}\left(e^{i \theta_{0}}\right)-$ $\varphi_{p}\left(e^{i \theta_{0}}\right)=0$. Then, either $\mu^{(p)}$ has no pure point in the gap or else it has a pure point at $z=e^{i \theta_{0}}$.

The results on measures with associated sequence of periodic Verblunsky coefficients presented above will be used in Sects. 4 and 5.

\section{On measures associated with alternating sign sequences $\left\{c_{n}\right\}$}

First, we provide three lemmas that will be useful to derive the subsequent results.

Lemma 1 Let $\mathcal{W}_{n}(x)$ satisfying (3) and $R_{n}(z)$ satisfying (1). Then, for $c \in \mathbb{R}$, the following statements are equivalent: 
(i) $c_{n}=(-1)^{n} c, n \geq 1$;

(ii) for $n \geq 0, R_{2 n}(z)$ has real coefficients and $R_{2 n+1}(z)=[(1-i c) z+(1+i c)] \widetilde{R}_{2 n}(z)$, where $\widetilde{R}_{0}(x)=1$ and $\widetilde{R}_{2 n}(z)$ is also a polynomial with real coefficients;

(iii) for $n \geq 0, \mathcal{W}_{2 n}(x)$ is an even polynomial of degree $2 n$ and

$$
\mathcal{W}_{2 n+1}(x)=\left(x+c \sqrt{1-x^{2}}\right) \widetilde{\mathcal{W}}_{2 n}(x)
$$

with $\widetilde{\mathcal{W}}_{0}(x)=1$ and $\widetilde{\mathcal{W}}_{2 n}(x)$ an even polynomial of degree $2 n$.

Proof (i) $\Rightarrow$ (ii) Since $R_{0}(z)=1$ and $R_{1}(z)=[(1-i c) z+(1+i c)] \widetilde{R}_{0}(z)$, with $\widetilde{R}_{0}(z)=1$, it follows that the result holds for $n=0$. Furthermore, if (ii) holds for $n=k \in \mathbb{N}$ then, from the three term recurrence relation (1), we obtain

$$
\begin{aligned}
R_{2(k+1)}(z) & =[(1+i c) z+(1-i c)][(1-i c) z+(1+i c)] \widetilde{R}_{2 k}(z)-4 d_{2 k+2} z R_{2 k}(z) \\
& =\left[\left(1+c^{2}\right) z^{2}+2\left(1-c^{2}\right) z+\left(1+c^{2}\right)\right] \widetilde{R}_{2 k}(z)-4 d_{2 k+2} z R_{2 k}(z) .
\end{aligned}
$$

Consequently, since we are assuming that $\widetilde{R}_{2 k}(z)$ and $R_{2 k}(z)$ are polynomials with real coefficients, we conclude that $R_{2(k+1)}(z)$ also has real coefficients. Moreover, using again (1), we can see that

$$
\begin{aligned}
R_{2(k+1)+1}(z) & =[(1-i c) z+(1+i c)] R_{2(k+1)}(z)-4 d_{2 k+3} z[(1-i c) z+(1+i c)] \widetilde{R}_{2 k}(z) \\
& =[(1-i c) z+(1+i c)] \widetilde{R}_{2(k+1)}(z),
\end{aligned}
$$

where $\widetilde{R}_{2(k+1)}(z)=R_{2(k+1)}(z)-4 d_{2 k+3} z \widetilde{R}_{2 k}(z)$ is also a polynomial with real coefficients, once $R_{2(k+1)}(z)$ and $\widetilde{R}_{2 k}(z)$ have real coefficients. Therefore, using mathematical induction, we conclude that the statement (ii) holds for all $n \geq 0$.

(ii) $\Rightarrow$ (iii) By (ii), $R_{2 n}(z)$ has real coefficients for $n \geq 0$. Moreover, from the three term recurrence relation (1) we have that $R_{2 n}(z)$ is a self-inversive polynomial, i.e., $R_{2 n}^{*}(z)=z^{2 n} \overline{R_{2 n}(1 / \bar{z})}=R_{2 n}(z)$. Therefore, it follows (see Bracciali et al. 2016, Lemma $2.1)$ that $W_{2 n}(x)$ is an even polynomial of degree $2 n$ in the variable $x=\cos (\theta / 2)$. Similarly, since $\widetilde{R}_{2 n}(z)$ is also a self-inversive polynomial with real coefficients, we have that $\widetilde{\mathcal{W}}_{2 n}(x)=\left(4 e^{i \theta}\right)^{-n} \widetilde{R}_{2 n}\left(e^{i \theta}\right)$ is an even polynomial of degree $2 n$. Then, since $R_{2 n+1}(z)=[(1-i c) z+(1+i c)] \widetilde{R}_{2 n}(z)$, from the relation (2) it follows that $\mathcal{W}_{2 n+1}(x)=$ $\left(x+c \sqrt{1-x^{2}}\right) \widetilde{\mathcal{W}}_{2 n}(x)$.

(iii) $\Rightarrow$ (i) Using the assumption (iii) and the three term recurrence relation (3), we have, for $s \geq 1$,

$$
\mathcal{W}_{2 s}(x)=\left(x-c_{2 s} \sqrt{1-x^{2}}\right)\left(x+c \sqrt{1-x^{2}}\right) \widetilde{\mathcal{W}}_{2 s-2}(x)-d_{2 s} \mathcal{W}_{2 s-2}(x)
$$

and

$$
\left(x+c \sqrt{1-x^{2}}\right) \widetilde{\mathcal{W}}_{2 s}(x)=\left(x-c_{2 s+1} \sqrt{1-x^{2}}\right) \mathcal{W}_{2 s}(x)-d_{2 s+1}\left(x+c \sqrt{1-x^{2}}\right) \widetilde{\mathcal{W}}_{2 s-2}(x) .
$$

Hence, since $\mathcal{W}_{2 s}(x), \mathcal{W}_{2 s-2}(x), \widetilde{\mathcal{W}}_{2 s}(x)$ and $\widetilde{\mathcal{W}}_{2 s-2}(x)$ are even polynomials, and we can use the relations (9) and (10) to conclude that $c_{2 s}=c$ and $c_{2 s+1}=-c$, for $s \geq 1$. Moreover, using the assumption (iii) and the definition of $\mathcal{W}_{1}(x)$ it is easy to see that $c_{1}=-c$.

Consider now the polynomials $\hat{R}_{n}(z)$ satisfying

$$
\hat{R}_{n+1}(z)=\left[\left(1+i \hat{c}_{n+1}\right) z+\left(1-i \hat{c}_{n+1}\right)\right] \hat{R}_{n}(z)-4 d_{n+1} z \hat{R}_{n-1}(z), \quad n \geq 1,
$$

with $\hat{R}_{0}(z)=1, \hat{R}_{1}(z)=\left(1+i \hat{c}_{1}\right) z+\left(1-i \hat{c}_{1}\right)$ and $\hat{c}_{n}=-c_{n}$.

The following lemma gives the relation between the polynomials $R_{n}(z)$ and $\hat{R}_{n}(z)$. 
Lemma 2 Let $R_{n}(z)$ satisfying (1) and $\hat{R}_{n}(z)$ satisfying (11). Then, the following holds:

$$
R_{n}(z)=\overline{\hat{R}_{n}(\bar{z})}, \quad n=0,1,2, \ldots .
$$

Proof Clearly, the result holds for $n=0$ and $n=1$. Suppose that the result holds for $n=0,1, \ldots, k$. Then, from the recurrence relation (11), we have

$$
\begin{aligned}
R_{k+1}(z) & =\left[\left(1+i c_{k+1}\right) z+\left(1-i c_{k+1}\right)\right] R_{k}(z)-4 d_{k+1} z R_{k-1}(z) \\
& =\left[\left(1+i c_{k+1}\right) z+\left(1-i c_{k+1}\right)\right] \overline{\hat{R}_{k}(\bar{z})}-4 d_{k+1} z{\hat{R_{k-1}}}_{k}(\bar{z}) \\
& =\overline{\hat{R}_{k+1}(\bar{z})} .
\end{aligned}
$$

Now, the result follows by mathematical induction.

Observe that the Lemma 2 provides also a relation between the zeros of the polynomials $R_{n}(z)$ and the zeros of $\hat{R}_{n}(z)$, namely, if $z_{n, j}$ is a zero of $R_{n}(z)$, then $\bar{z}_{n, j}$ is a zero of $\hat{R}_{n}(z)$.

Denoting by $\operatorname{sgn}(f(a))$ the sign of a certain function $f$ at a point $a$ of its domain, we can establish the following result.

Lemma 3 Let $\mathcal{W}_{n}(x)$ satisfying (3), where $\left\{d_{n}\right\}_{n=1}^{\infty}$ is a positive chain sequence and $c_{n}=$ $(-1)^{n} \tilde{c}_{n}$, with $\tilde{c}_{n} \geq c>0$, for $n \geq 1$. If $\varepsilon$ is a real number satisfying $0<\varepsilon<c$, then

$$
\operatorname{sgn}\left(\mathcal{W}_{n}\left(x_{j}^{(\varepsilon)}\right)\right)=(-1)^{\lfloor n / 2\rfloor}, \quad j \in\{0,1\}, \quad n \geq 0,
$$

where $x_{0}^{(\varepsilon)}=-c_{\varepsilon} / \sqrt{1+c_{\varepsilon}^{2}}$ and $x_{1}^{(\varepsilon)}=c_{\varepsilon} / \sqrt{1+c_{\varepsilon}^{2}}$, with $c_{\varepsilon}=c-\varepsilon$.

Proof Since $\mathcal{W}_{0}(x)=1$, the result clearly occurs for $n=0$. If $\varepsilon$ is a real number such that $0<\varepsilon<c$ and $c_{\varepsilon}=c-\varepsilon$, then, for $n \geq 1$ we have $\tilde{c}_{n} \geq c>c_{\varepsilon}>0$. Moreover, by considering $x_{0}^{(\varepsilon)}=-c_{\varepsilon} / \sqrt{1+c_{\varepsilon}^{2}}$ and $x_{1}^{(\varepsilon)}=c_{\varepsilon} / \sqrt{1+c_{\varepsilon}^{2}}$, we can show that, for $n \geq 1$,

$$
\operatorname{sgn}\left(x_{0}^{(\varepsilon)}-\tilde{c}_{n} \sqrt{1-\left[x_{0}^{(\varepsilon)}\right]^{2}}\right)=\operatorname{sgn}\left(x_{1}^{(\varepsilon)}-\tilde{c}_{n} \sqrt{1-\left[x_{1}^{(\varepsilon)}\right]^{2}}\right)=-1
$$

and

$$
\operatorname{sgn}\left(x_{0}^{(\varepsilon)}+\tilde{c}_{n} \sqrt{1-\left[x_{0}^{(\varepsilon)}\right]^{2}}\right)=\operatorname{sgn}\left(x_{1}^{(\varepsilon)}+\tilde{c}_{n} \sqrt{1-\left[x_{1}^{(\varepsilon)}\right]^{2}}\right)=1 .
$$

Now, suppose that the relation (12) holds for $n=1,2, \ldots, k$. Then, if $k=2 s+1, s \geq 0$, we have

$$
\operatorname{sgn}\left(\mathcal{W}_{2 s+1}\left(x_{j}^{(\varepsilon)}\right)\right)=(-1)^{s}=\operatorname{sgn}\left(\mathcal{W}_{2 s}\left(x_{j}^{(\varepsilon)}\right)\right), \quad j \in\{0,1\} .
$$

Furthermore, using the three term recurrence relation (3), we obtain

$$
\mathcal{W}_{k+1}\left(x_{j}^{(\varepsilon)}\right)=\left(x_{j}^{(\varepsilon)}-\tilde{c}_{2 s+2} \sqrt{1-\left[x_{j}^{(\varepsilon)}\right]^{2}}\right) \mathcal{W}_{2 s+1}\left(x_{j}^{(\varepsilon)}\right)-d_{2 s+2} \mathcal{W}_{2 s}\left(x_{j}^{(\varepsilon)}\right), \quad j \in\{0,1\} .
$$

Since $d_{2 s+2}$ is an element of the positive chain sequence $\left\{d_{n}\right\}_{n=1}^{\infty}$, we obviously have $d_{2 s+2}>$ 0 . Thus, we can use (13), (15) and (16), to conclude that

$$
\operatorname{sgn}\left(\mathcal{W}_{k+1}\left(x_{j}^{(\varepsilon)}\right)\right)=-\operatorname{sgn}\left(\mathcal{W}_{2 s+1}\left(x_{j}^{(\varepsilon)}\right)\right)=(-1)^{s+1}=(-1)^{\lfloor(k+1) / 2\rfloor}, \quad j \in\{0,1\} .
$$

Similarly, if $k=2 s+2, s \geq 0$, then

$$
\operatorname{sgn}\left(\mathcal{W}_{2 s+2}\left(x_{j}^{(\varepsilon)}\right)\right)=(-1)^{s+1}=-\operatorname{sgn}\left(\mathcal{W}_{2 s+1}\left(x_{j}^{(\varepsilon)}\right)\right), \quad j \in\{0,1\} .
$$


On the other hand, using again the three term recurrence relation (3), we find

$$
\mathcal{W}_{k+1}\left(x_{j}^{(\varepsilon)}\right)=\left(x_{j}^{(\varepsilon)}+\tilde{c}_{2 s+3} \sqrt{1-\left[x_{j}^{(\varepsilon)}\right]^{2}}\right) \mathcal{W}_{2 s+2}\left(x_{j}^{(\varepsilon)}\right)-d_{2 s+3} \mathcal{W}_{2 s+1}\left(x_{j}^{(\varepsilon)}\right), \quad j \in\{0,1\} .
$$

Moreover, since $d_{2 s+3}>0$, from the relations (14), (17) and (18), we can see that

$$
\operatorname{sgn}\left(\mathcal{W}_{k+1}\left(x_{j}^{(\varepsilon)}\right)\right)=\operatorname{sgn}\left(\mathcal{W}_{2 s+2}\left(x_{j}^{(\varepsilon)}\right)\right)=(-1)^{s+1}=(-1)^{\lfloor(k+1) / 2\rfloor}, \quad j \in\{0,1\} .
$$

Therefore, using mathematical induction, we conclude that the property (12) holds for all $n \geq 0$.

Now, we consider the problem of giving estimates for the support of measures whose sequences $\left\{c_{n}\right\}_{n=1}^{\infty}$ are of alternating sign. We start with the case $c_{n}=(-1)^{n} c$, where $c \in \mathbb{R}$.

Let $\mathcal{C}_{1}$ and $\mathcal{C}_{2}$ be closed arcs on the unit circle given by

$$
\mathcal{C}_{1}=\left\{z \in \mathbb{T}: 0 \leq \arg (z) \leq \theta_{c}\right\} \quad \text { and } \mathcal{C}_{2}=\left\{z \in \mathbb{T}: 2 \pi-\theta_{c} \leq \arg (z) \leq 2 \pi\right\},
$$

where $\theta_{c}=\arccos \left(\frac{c^{2}-1}{c^{2}+1}\right) \in[0, \pi]$. Then, we can state the following.

Theorem 8 Let $\mu$ be the probability measure on the unit circle associated with the pair of sequences $\left\{\left\{c_{n}\right\}_{n=1}^{\infty},\left\{d_{n}\right\}_{n=1}^{\infty}\right\}$ where $c_{n}=(-1)^{n} c, c \in \mathbb{R}$ and $\left\{d_{n}\right\}_{n=1}^{\infty}$ is a positive chain sequence. Then, the support of $\mu$ lies within $\mathcal{C}_{1} \cup \mathcal{C}_{2}$.

Proof Without loss of generality, we assume that $c \geq 0$. Consider the polynomials $R_{n}(z)$ given by (1). If we show that all zeros of $R_{n}(z)$ lie on $\mathcal{C}_{1} \cup \mathcal{C}_{2}$, then from Theorem 3 we obtain the desired result. To show this, we use the functions $\mathcal{W}_{n}(x)$ defined in (3) which are associated with the polynomials $R_{n}(z)$.

By Lemma 1, we have that $\mathcal{W}_{2 n+1}(x)=\left(x+c \sqrt{1-x^{2}}\right) \widetilde{\mathcal{W}}_{2 n}(x)$ with $\widetilde{\mathcal{W}}_{2 n}(x)$ an even polynomial of degree $2 n$. Moreover, $\mathcal{W}_{2 n}(x)$ is also an even polynomial of degree $2 n$. This means that $\frac{-c}{\sqrt{1+c^{2}}}$ is always a zero of $\mathcal{W}_{2 n+1}(x)$ and the other $2 n$ zeros of these functions have a symmetry about the origin. Likewise, $\mathcal{W}_{2 n}(x)$ being an even polynomial, all of their zeros are symmetric with respect to the origin.

Therefore, from the symmetry of the zeros observed above and taking into account the interlacing property (4), it follows that all zeros of $\mathcal{W}_{n}(x)$ lie in $\left(-1, \frac{-c}{\sqrt{1+c^{2}}}\right] \cup\left[\frac{c}{\sqrt{1+c^{2}}}, 1\right)$.

Finally, if we denote the zeros of $\mathcal{W}_{n}(x)$ by $x_{n, j}$ and the zeros of $R_{n}(z)$ by $z_{n, j}$, then they are related by $x_{n, j}=\cos \left(\frac{\theta_{n, j}}{2}\right)$ where $z_{n, j}=e^{i \theta_{n, j}}, j=1,2, \ldots, n$. This shows that $R_{n}(z)$ has all of its zeros on $\mathcal{C}_{1} \cup \mathcal{C}_{2}$.

Notice that Theorem 8 leads to an estimative for the support of the measure in the case where $c_{n}=(-1)^{n} \tilde{c}_{n}$ and $\tilde{c}_{n}$ is a constant sequence. We use this initial estimative as motivation to obtain a more general result.

Theorem 9 Let $\mu$ be the probability measure on the unit circle associated with the pair of sequences $\left\{\left\{c_{n}\right\}_{n=1}^{\infty},\left\{d_{n}\right\}_{n=1}^{\infty}\right\}$, where $c_{n}=(-1)^{n} \tilde{c}_{n}, \tilde{c}_{n} \geq c>0$ and $\left\{d_{n}\right\}_{n=1}^{\infty}$ is a positive chain sequence. Then, the support of $\mu$ lies within $\mathcal{C}_{1} \cup \mathcal{C}_{2}$.

Proof As in the proof of the previous theorem, it is enough to show that all the $n$ zeros of $R_{n}(z), n \geq 1$, lie on $\mathcal{C}_{1} \cup \mathcal{C}_{2}$, or equivalently from the relation (2), just prove that all $n$ zeros, in $(-1,1)$, of the functions $\mathcal{W}_{n}(x), n \geq 1$, given by (3), belong to the set $X=$ $\left(-1,-c / \sqrt{1+c^{2}}\right] \cup\left[c / \sqrt{1+c^{2}}, 1\right)$. 
Let $\varepsilon$ be a real number such that $0<\varepsilon<c$, and consider the numbers $x_{0}^{(\varepsilon)}=-c_{\varepsilon} / \sqrt{1+c_{\varepsilon}^{2}}$ and $x_{1}^{(\varepsilon)}=c_{\varepsilon} / \sqrt{1+c_{\varepsilon}^{2}}$, where $c_{\varepsilon}=c-\varepsilon$.

Initially, we will show that all the $n$ zeros of $\mathcal{W}_{n}(x)$, in $(-1,1)$, belong to the set $X^{(\varepsilon)}=$ $\left(-1, x_{0}^{(\varepsilon)}\right] \cup\left[x_{1}^{(\varepsilon)}, 1\right)$. To do this, we will use mathematical induction on $n$.

Notice that $x_{1,1}=-\tilde{c}_{1} / \sqrt{1+\tilde{c}_{1}^{2}}$ is the only zero of $\mathcal{W}_{1}(x)=x+\tilde{c}_{1} \sqrt{1-x^{2}}$ in $(-1,1)$. Therefore, since $\tilde{c}_{1} \geq c>c_{\varepsilon}$, we have that $x_{1,1} \in X^{(\varepsilon)}$. Consequently, the result is valid for $n=1$.

Now, suppose that all the $k$ zeros, $k \in \mathbb{N}$, of $\mathcal{W}_{k}(x)$, in $(-1,1)$, belong to $X^{(\varepsilon)}$. We claim that $\mathcal{W}_{k+1}(x)$ has no zeros in the interval $Y^{(\varepsilon)}=(-1,1) \backslash X^{(\varepsilon)}=\left(x_{0}^{(\varepsilon)}, x_{1}^{(\varepsilon)}\right)$. Indeed, from Lemma 3, we have $\operatorname{sign}\left(\mathcal{W}_{k+1}\left(x_{0}^{(\varepsilon)}\right)\right)=\operatorname{sign}\left(\mathcal{W}_{k+1}\left(x_{1}^{(\varepsilon)}\right)\right)$. Hence, if we assume that there exists at least one zero of $\mathcal{W}_{k+1}(x)$ inside the interval $Y^{(\varepsilon)}$ then, $\operatorname{since} \operatorname{sign}\left(\mathcal{W}_{k+1}\left(x_{0}^{(\varepsilon)}\right)\right)=$ $\operatorname{sign}\left(\mathcal{W}_{k+1}\left(x_{1}^{(\varepsilon)}\right)\right)$, we conclude that $\mathcal{W}_{k+1}(x)$ has at least two zeros in $Y^{(\varepsilon)}$. Consequently, using the interlacing property for the zeros of $\mathcal{W}_{k+1}(x)$ and $\mathcal{W}_{k}(x)$, given in (4), we should have, at least, one zero of $\mathcal{W}_{k}(x)$ inside the interval $Y^{(\varepsilon)}$. But this cannot happen because all the zeros of $\mathcal{W}_{k}(x)$, in $(-1,1)$, belong to $X^{(\varepsilon)}$. Therefore, using mathematical induction, we conclude that all the zeros of $\mathcal{W}_{n}(x)$ belong to $X^{(\varepsilon)}$ for all $n \geq 1$.

Finally, by letting $\varepsilon \rightarrow 0$, we see that $\mathcal{W}_{n}(x)$ has all its $n$ zeros in $X$, which completes the proof of the theorem.

Corollary 1 Let $\mu$ be the probability measure on the unit circle associated with the pair of sequences $\left\{\left\{c_{n}\right\}_{n=1}^{\infty},\left\{d_{n}\right\}_{n=1}^{\infty}\right\}$, where $c_{n}=(-1)^{n} \tilde{c}_{n}, \tilde{c}_{n} \leq c<0$ and $\left\{d_{n}\right\}_{n=1}^{\infty}$ is a positive chain sequence. Then, the support of $\mu$ lies on $\mathcal{C}_{1} \cup \mathcal{C}_{2}$.

Proof First, one can observe that $-c_{n}=(-1)^{n}\left(-\tilde{c}_{n}\right)$, with $-\tilde{c}_{n} \geq-c>0$. Hence, if $\hat{\mu}$ is the probability measure associated with the pair $\left\{\left\{-c_{n}\right\}_{n=1}^{\infty},\left\{d_{n}\right\}_{n=1}^{\infty}\right\}$, from Theorem 9 it follows that $\hat{R}_{n}(z)$ given by (11) has all zeros on $\mathcal{C}_{1} \cup \mathcal{C}_{2}$ and that $\hat{\mu}$ has its support within $\mathcal{C}_{1} \cup \mathcal{C}_{2}$. Now, the result is an immediate consequence of Lemma 2.

Let $\mu$ be the measure associated with the pair $\left\{\left\{c_{n}\right\}_{n=1}^{\infty},\left\{d_{n}\right\}_{n=1}^{\infty}\right\}$, where $\left\{c_{n}\right\}_{n=1}^{\infty}$ satisfies the condition $c_{2 n}=-c_{2 n-1}, n \geq 1$.

Starting from $\mu$ we desire to get a new measure $\tilde{\mu}$ associated with the pair of real sequences $\left\{\left\{\tilde{c}_{n}\right\}_{n=1}^{\infty},\left\{\tilde{d}_{n}\right\}_{n=1}^{\infty}\right\}$, where $\left\{\tilde{c}_{n}\right\}_{n=1}^{\infty}$ must satisfy the condition $\tilde{c}_{2 n}=\tilde{c}_{2 n-1}=c_{2 n}, n \geq 1$.

Let us consider the sequence of complex numbers $\left\{\beta_{n}\right\}_{n=1}^{\infty}$ given by

$$
\beta_{n}=-\left(\frac{1+i c_{2 n}}{1-i c_{2 n}}\right), n=1,2, \ldots
$$

The next theorem shows how to get the required measure $\tilde{\mu}$ from the measure $\mu$.

Theorem 10 Let $\mu$ be the probability measure on the unit circle associated with the pair of sequences $\left\{\left\{c_{n}\right\}_{n=1}^{\infty},\left\{d_{n}\right\}_{n=1}^{\infty}\right\}$, where $c_{2 n}=-c_{2 n-1}, n \geq 1$. Let $\left\{\beta_{n}\right\}_{n=1}^{\infty}$ be the sequence of complex numbers defined by (19). In addition, let $\tilde{\mu}$ be the measure associated with the sequence of Verblunsky coefficients $\left\{\tilde{\alpha}_{n}\right\}_{n=0}^{\infty}$ given by

$$
\tilde{\alpha}_{2 n+1}=\left(\prod_{j=1}^{n+1} \beta_{j}^{2}\right) \alpha_{2 n+1} \quad \text { and } \quad \tilde{\alpha}_{2 n}=\left(\prod_{j=1}^{n} \beta_{j}^{2}\right) \beta_{n+1} \alpha_{2 n}, \quad n=0,1,2, \ldots,
$$


where $\left\{\alpha_{n}\right\}_{n=0}^{\infty}$ is the sequence of Verblunsky coefficients related to $\mu$. If $\left\{\left\{\tilde{c}_{n}\right\}_{n=1}^{\infty},\left\{\tilde{d}_{n}\right\}_{n=1}^{\infty}\right\}$ is the pair of sequences associated with the measure $\tilde{\mu}$ and if $\left\{\tilde{m}_{n}\right\}_{n=0}^{\infty}$ is the minimal parameter sequence for $\left\{\tilde{d}_{n}\right\}_{n=1}^{\infty}$, then the following holds

$$
\tilde{c}_{2 n}=\tilde{c}_{2 n-1}=c_{2 n}, \quad \tilde{m}_{2 n-1}=1-m_{2 n-1} \quad \text { and } \tilde{m}_{2 n}=m_{2 n}, \quad n=1,2, \ldots .
$$

Proof Using the assumption $c_{2 n}=-c_{2 n-1}$, we obtain

$$
\tau_{2 n}=1 \quad \text { and } \quad \tau_{2 n+1}=\frac{1+i c_{2 n+2}}{1-i c_{2 n+2}}, \quad n=0,1, \ldots .
$$

Hence, from (5) and (21), we have

$$
\alpha_{2 n}=\frac{1-2 m_{2 n+1}+i c_{2 n+2}}{1+i c_{2 n+2}} \text { and } \quad \alpha_{2 n+1}=\frac{1-2 m_{2 n+2}-i c_{2 n+2}}{1+i c_{2 n+2}}, \quad n=0,1, \ldots
$$

Now, let $\left\{\hat{m}_{n}\right\}_{n=0}^{\infty}$ be the minimal parameter sequence for a positive chain sequence $\left\{\hat{d}_{n}\right\}_{n=1}^{\infty}$ and $\left\{\hat{\alpha}_{n}\right\}_{n=0}^{\infty}$ the sequence of Verblunsky coefficients of a probability measure on the unit circle, $\hat{\mu}$, associated with the pair of real sequences $\left\{\left\{\hat{c}_{n}\right\}_{n=1}^{\infty},\left\{\hat{d}_{n}\right\}_{n=1}^{\infty}\right\}$, where

$$
\hat{c}_{2 n}=\hat{c}_{2 n-1}=c_{2 n}, \quad \hat{m}_{2 n-1}=1-m_{2 n-1} \quad \text { and } \quad \hat{m}_{2 n}=m_{2 n}, \quad n=1,2, \ldots .
$$

Using the relations (5), (19), (20), (22) and (23), one can see that for $n \geq 0$,

$$
\begin{aligned}
\hat{\alpha}_{2 n+1} & =\left(\frac{1+i \hat{c}_{2 n+1}}{1-i \hat{c}_{2 n+1}}\right)\left(\prod_{k=1}^{2 n} \frac{1+i \hat{c}_{k}}{1-i \hat{c}_{k}}\right)\left[\frac{1-2 \hat{m}_{2 n+2}-i \hat{c}_{2 n+2}}{1-i \hat{c}_{2 n+2}}\right] \\
& =\left(\frac{1+i c_{2 n+2}}{1-i c_{2 n+2}}\right)^{2}\left[\prod_{j=1}^{n}\left(\frac{1+i c_{2 j}}{1-i c_{2 j}}\right)^{2}\right]\left[\frac{1-2 m_{2 n+2}-i c_{2 n+2}}{1+i c_{2 n+2}}\right] \\
& =\left(\prod_{j=1}^{n+1} \beta_{j}^{2}\right) \alpha_{2 n+1}=\tilde{\alpha}_{2 n+1} .
\end{aligned}
$$

Similarly, using again (5), (19), (20), (22) and (23), we obtain for $n \geq 0$,

$$
\begin{aligned}
\hat{\alpha}_{2 n} & =\left(\prod_{k=1}^{2 n} \frac{1+i \hat{c}_{k}}{1-i \hat{c}_{k}}\right)\left[\frac{1-2 \hat{m}_{2 n+1}-i \hat{c}_{2 n+1}}{1-i \hat{c}_{2 n+1}}\right] \\
& =\left[\prod_{j=1}^{n}\left(\frac{1+i c_{2 j}}{1-i c_{2 j}}\right)^{2}\right]\left[-\left(\frac{1+i c_{2 n+2}}{1-i c_{2 n+2}}\right)\right]\left[\frac{1-2 m_{2 n+1}+i c_{2 n+2}}{1+i c_{2 n+2}}\right] \\
& =\left(\prod_{j=1}^{n} \beta_{j}{ }^{2}\right) \beta_{n+1} \alpha_{2 n}=\tilde{\alpha}_{2 n} .
\end{aligned}
$$

Thus, $\tilde{\alpha}_{n}=\hat{\alpha}_{n}$ for $n \geq 0$ and, consequently, $\tilde{\mu}=\hat{\mu}$. Hence, from the uniqueness of the pair $\left\{\left\{\tilde{c}_{n}\right\}_{n=1}^{\infty},\left\{\tilde{d}_{n}\right\}_{n=1}^{\infty}\right\}$ given by Theorem 1, we have $\tilde{m}_{0}=\hat{m}_{0}=0$,

$$
\tilde{c}_{n}=\hat{c}_{n} \quad \text { and } \quad \tilde{m}_{n}=\hat{m}_{n}, \quad n=1,2, \ldots,
$$

which completes the proof of the theorem. 
Corollary 2 Let $\mu$ be the probability measure on the unit circle associated with the pair of sequences $\left\{\left\{c_{n}\right\}_{n=1}^{\infty},\left\{d_{n}\right\}_{n=1}^{\infty}\right\}$, where $c_{n}=(-1)^{n} c, n \geq 1$ and $c \in \mathbb{R}$. In addition, let $\beta=-\left(\frac{1+i c}{1-i c}\right)$ and $\tilde{\mu}(z)=\mu(\beta z)$ the measure associated with the pair $\left\{\left\{\tilde{c}_{n}\right\}_{n=1}^{\infty},\left\{\tilde{d}_{n}\right\}_{n=1}^{\infty}\right\}$. Then, for $n \geq 1, \tilde{c}_{n}=c$.

Proof First, notice that if $\tilde{\mu}(z)=\mu(\beta z)$, the corresponding Verblunsky coefficients are related by $\tilde{\alpha}_{n}=\beta^{n+1} \alpha_{n}, n \geq 0$ (see, for example, Costa et al. 2013). Hence, the result follows from Theorem 10 with $c_{2 n}=c, n \geq 1$.

\section{Measures associated with periodic Verblunsky coefficients}

The first theorem in this section gives a characterization of measures with associated sequence of periodic Verblunsky coefficients in terms of the pair of real sequences $\left\{\left\{c_{n}\right\}_{n=1}^{\infty},\left\{d_{n}\right\}_{n=1}^{\infty}\right\}$, where $\left\{d_{n}\right\}_{n=1}^{\infty}$ is a positive chain sequence. Throughout this section $b_{n}=1-2 m_{n}, n \geq 1$, where $\left\{m_{n}\right\}_{n=0}^{\infty}$ is the minimal parameter sequence of $\left\{d_{n}\right\}_{n=1}^{\infty}$.

Theorem 11 Let $\mu$ be the probability measure on the unit circle associated with the pair of sequences $\left\{\left\{c_{n}\right\}_{n=1}^{\infty},\left\{d_{n}\right\}_{n=1}^{\infty}\right\}$. Then, the measure $\mu$ has associated sequence of periodic Verblunsky coefficients $\left\{\alpha_{n}\right\}_{n=0}^{\infty}$ of period $p$ if, and only if, for $n \geq 0$,

$$
\begin{aligned}
\sum_{j=n+1}^{n+p} \arg \left(\frac{1+i c_{j}}{1-i c_{j}}\right)= & \arg \left(\frac{b_{n+1}-i c_{n+1}}{1-i c_{n+1}}\right)-\arg \left(\frac{b_{n+p+1}-i c_{n+p+1}}{1-i c_{n+p+1}}\right) \\
& +2 k_{n} \pi, k_{n} \in \mathbb{Z}
\end{aligned}
$$

and

$$
\frac{b_{n+1}^{2}+c_{n+1}^{2}}{1+c_{n+1}^{2}}=\frac{b_{n+p+1}^{2}+c_{n+p+1}^{2}}{1+c_{n+p+1}^{2}} .
$$

Proof First one can observe, from (5), that for $n \geq 0$,

$$
\begin{aligned}
\alpha_{n+p}=\alpha_{n} & \Leftrightarrow \bar{\tau}_{n+p}\left[\frac{b_{n+p+1}-i c_{n+p+1}}{1-i c_{n+p+1}}\right]=\bar{\tau}_{n}\left[\frac{b_{n+1}-i c_{n+1}}{1-i c_{n+1}}\right] \\
& \Leftrightarrow\left(\prod_{j=n+1}^{n+p} \frac{1+i c_{j}}{1-i c_{j}}\right)\left[\frac{b_{n+p+1}-i c_{n+p+1}}{1-i c_{n+p+1}}\right]=\left[\frac{b_{n+1}-i c_{n+1}}{1-i c_{n+1}}\right] .
\end{aligned}
$$

Now the result follows by comparing, respectively, the modulus and the argument of the numbers

$$
\left(\prod_{j=n+1}^{n+p} \frac{1+i c_{j}}{1-i c_{j}}\right)\left[\frac{b_{n+p+1}-i c_{n+p+1}}{1-i c_{n+p+1}}\right] \text { and }\left[\frac{b_{n+1}-i c_{n+1}}{1-i c_{n+1}}\right], n \geq 0 .
$$

We say that $\mu$ is a symmetric measure if $d \mu(z)=-d \mu(1 / z), z \in \mathbb{T}$. From the results established in Castillo et al. (2014), one can observe that $\mu$ is symmetric if and only if $c_{n}=0$, $n \geq 1$, with $\left\{c_{n}\right\}_{n=1}^{\infty}$ given as in Theorem 1. Thus, as a consequence of Theorem 11, we have the following result. 
Corollary 3 Let $\mu$ be the probability measure on the unit circle associated with the pair of sequences $\left\{\left\{c_{n}\right\}_{n=1}^{\infty},\left\{d_{n}\right\}_{n=1}^{\infty}\right\}$, where $\left\{c_{n}\right\}_{n=1}^{\infty}$ and $\left\{m_{n}\right\}_{n=1}^{\infty}$ are periodic sequences of period $p$. In addition, suppose that $c_{2 n}=-c_{2 n-1}, n \geq 1$. Then,

(i) if $p$ is even, the measure $\mu$ has associated $p$-periodic sequence of Verblunsky coefficients;

(ii) if $p$ is odd, the measure $\mu$ is symmetric and has associated $p$-periodic sequence of Verblunsky coefficients.

Proof (i) Clearly, we have that (24) and (25) hold. Hence, the result follows by Theorem 11.

(ii) If $p$ is odd, using the periodicity of $\left\{c_{n}\right\}_{n=1}^{\infty}$ and the assumption that $c_{2 n}=-c_{2 n-1}$, we conclude that $c_{n}=0, n \geq 1$. Hence, $\mu$ is symmetric. Moreover, since $\left\{m_{n}\right\}_{n=1}^{\infty}$ is a periodic sequence of period $p$ and $c_{n}=0, n \geq 1$, the conditions (24) and (25) of Theorem 11 can be easily verified. Consequently, the measure $\mu$ has associated $p$-periodic sequence of Verblunsky coefficients.

The Corollary 3 shows that if we choose the sequence $\left\{c_{n}\right\}_{n=1}^{\infty} p$-periodic ( $p$ even) and such that $c_{2 n}=-c_{2 n-1}$, then it is possible, by choosing $\left\{m_{n}\right\}_{n=1}^{\infty}$ also $p$-periodic, to get a measure $\mu^{(p)}$ whose Verblunsky coefficients are periodic with the same period. Notice that in the case when $c_{2 n}=-c_{2 n-1}$ and $c_{2 n}>0$ (or $c_{2 n}<0$ ) for $n \geq 1$, the sequence $\left\{c_{n}\right\}_{n=1}^{\infty}$ has the alternating sign property.

The next theorem provides a geometric characterization for the choice of $\left\{c_{n}\right\}_{n=1}^{\infty}$ and $\left\{m_{n}\right\}_{n=1}^{\infty}$ considered above.

Theorem 12 Let $p$ be an even natural number and $\mu^{(p)}$ be the probability measure associated with the pair $\left\{\left\{c_{n}\right\}_{n=1}^{\infty},\left\{d_{n}\right\}_{n=1}^{\infty}\right\}$. Then, the following statements are equivalent:

(i) The sequences $\left\{c_{n}\right\}_{n=1}^{\infty}$ and $\left\{m_{n}\right\}_{n=1}^{\infty}$ are $p$-periodic with $c_{2 n}=-c_{2 n-1}, n \geq 1$.

(ii) The sequence of Verblunsky coefficients $\left\{\alpha_{n}\right\}_{n=0}^{\infty}$ associated with the measure $\mu^{(p)}$ is $p-$ periodic. In addition, for $k \in\left\{0,1, \ldots, \frac{p-2}{2}\right\}$, the straight lines connecting $\alpha_{2 k}$ to 1 and $\alpha_{2 k+1}$ to -1 are parallel.

Proof (i) $\Rightarrow$ (ii) From Corollary 3, it is immediate that $\left\{\alpha_{n}\right\}_{n=0}^{\infty}$ is a periodic sequence with period $p$. On the other hand, by the assumption that $c_{2 n}=-c_{2 n-1}$ and by (5), for $n \geq 0$, we have

$$
\alpha_{2 n}=\frac{b_{2 n+1}+i c_{2 n+2}}{1+i c_{2 n+2}}=1+\lambda_{2 n}\left(-1-i c_{2 n+1}\right),
$$

where $\lambda_{2 n}=\frac{1-b_{2 n+1}}{1+c_{2 n+1}^{2}}$. Similarly, for $n \geq 0$,

$$
\alpha_{2 n+1}=\frac{b_{2 n+2}-i c_{2 n+2}}{1+i c_{2 n+2}}=-1+\lambda_{2 n+1}\left(-1+i c_{2 n+2}\right),
$$

where $\lambda_{2 n+1}=-\frac{1+b_{2 n+2}}{1+c_{2 n+2}^{2}}$.

Hence, for each $k \in\left\{0,1, \ldots, \frac{p-2}{2}\right\}$, one can see that $\alpha_{2 k} \in r_{2 k}$, where $r_{2 k}$ is the straight line with parametric equation given by $r_{2 k}(t)=1+t\left(-1-i c_{2 k+1}\right), t \in \mathbb{R}$.

Similarly, for each $k \in\left\{0,1, \ldots, \frac{p-2}{2}\right\}$, one can see that $\alpha_{2 k+1} \in r_{2 k+1}$, where $r_{2 k+1}$ is the straight line with parametric equation given by $r_{2 k+1}(t)=-1+t\left(-1+i c_{2 k+2}\right), t \in \mathbb{R}$.

Finally, since $-1-i c_{2 k+1}=-1+i c_{2 k+2}$ it follows that $r_{2 k} \| r_{2 k+1}$, for each $k \in$ $\left\{0,1, \ldots, \frac{p-2}{2}\right\}$. 
(ii) $\Rightarrow$ (i) Let $\alpha_{j}=x_{j}+i y_{j}, j=0,1, \ldots, p-1$. If $j=2 k, k \in\left\{0,1, \ldots, \frac{p-2}{2}\right\}$, we can write

$$
\alpha_{2 k}=1+\lambda_{2 k}\left(-1-i \tilde{c}_{2 k+1}\right)
$$

where

$$
\lambda_{2 k}=\frac{1-\tilde{b}_{2 k+1}}{1+\tilde{c}_{2 k+1}^{2}}, \quad \tilde{c}_{2 k+1}=\frac{y_{2 k}}{x_{2 k}-1} \quad \text { and } \quad \tilde{b}_{2 k+1}=1+\frac{\left(x_{2 k}-1\right)^{2}+y_{2 k}^{2}}{x_{2 k}-1} .
$$

Likewise, if $j=2 k+1, k \in\left\{0,1, \ldots, \frac{p-2}{2}\right\}$, we can write

$$
\alpha_{2 k+1}=-1+\lambda_{2 k+1}\left(-1+i \tilde{c}_{2 k+2}\right) \text {, }
$$

where

$$
\lambda_{2 k+1}=-\frac{1+\tilde{b}_{2 k+2}}{1+\tilde{c}_{2 k+2}^{2}}, \quad \tilde{c}_{2 k+2}=\frac{-y_{2 k+1}}{1+x_{2 k+1}} \quad \text { and } \quad \tilde{b}_{2 k+2}=-1+\frac{\left(1+x_{2 k+1}\right)^{2}+y_{2 k+1}^{2}}{1+x_{2 k+1}} .
$$

Hence, if we set $\tilde{b}_{n}=1-2 \tilde{m}_{n}$, from $\alpha_{n+p}=\alpha_{n}$, (27) and (29), one can see that

$$
\tilde{c}_{n+p}=\tilde{c}_{n} \text { and } \tilde{m}_{n+p}=\tilde{m}_{n}, \quad n=1,2, \ldots .
$$

For each $k \in\left\{0,1, \ldots, \frac{p-2}{2}\right\}$, let $r_{2 k}$ be the straight line connecting $\alpha_{2 k}$ to 1 and $r_{2 k+1}$ the straight line connecting $\alpha_{2 k+1}$ to -1 . Then, from (26), (28), (30) and since $r_{2 k} \| r_{2 k+1}, k \in$ $\left\{0,1, \ldots, \frac{p-2}{2}\right\}$, it follows that

$$
\tilde{c}_{2 n+2}=-\tilde{c}_{2 n+1}, \quad n=0,1, \ldots .
$$

Hence, from (26) to (31), we have, for $n \geq 0$,

$$
\alpha_{2 n}=\frac{\tilde{b}_{2 n+1}+i \tilde{c}_{2 n+2}}{1+i \tilde{c}_{2 n+2}} \text { and } \alpha_{2 n+1}=\frac{\tilde{b}_{2 n+2}-i \tilde{c}_{2 n+2}}{1+i \tilde{c}_{2 n+2}} .
$$

Finally, using the formula (5) for $\alpha_{n}$ and the relation (32) one can see, by mathematical induction, that for $n \geq 1$,

$$
\tilde{c}_{n}=c_{n} \text { and } \tilde{m}_{n}=m_{n}
$$

This completes the proof.

Observe that Theorem 12 shows that to choose a periodic sequence $\left\{\alpha_{n}\right\}$ of period $p$ ( $p$ even) with $\alpha_{j}$ on certain parallel straight lines is equivalent to choosing the sequences $\left\{c_{n}\right\}$ and $\left\{m_{n}\right\}$ also $p$-periodic with the additional property $c_{2 n+2}=-c_{2 n+1}, n \geq 0$. In Figs. 1 and 2 , we show some examples of possible choices for $\left\{c_{n}\right\}$ and $\left\{m_{n}\right\}$.

The following results deal with how to calculate (from the point of view of chain sequences) the pure points and the respective masses of a measure $\mu^{(p)}$, whose associated sequence of Verblunsky coefficients is periodic. In Simon (2005b), there is another approach to the same problem.

We begin with a lemma that leads to a characterization of the possible pure points (that we denote by $w$ ) of the measure $\mu^{(p)}$ in terms of the sequence $\left\{\tau_{n}(w)\right\}$ defined in (6).

Lemma 4 Let $\mu^{(p)}$ be a probability measure on the unit circle with associated $p$-periodic sequence of Verblunsky coefficients. Then, $w$ is a possible pure point of the measure $\mu^{(p)}$ if, and only if, the sequence $\left\{\tau_{n}(w)\right\}_{n=0}^{\infty}$ is periodic of period $p$. 
Fig. 1 Verblunsky coefficients associated with the choice $\left\{c_{n}\right\}=\{-c, c,-c, c, \ldots\}$ and $\left\{b_{n}\right\}=\left\{b_{1}, b_{2}, b_{1}, b_{2}, \ldots\right\}$, with $c>0$

Fig. 2 Verblunsky coefficients associated with the choice $\left\{c_{n}\right\}=$ $\left\{-c_{2}, c_{2},-c_{4}, c_{4},-c_{2}, c_{2}, \ldots\right\}$ and $\left\{b_{n}\right\}=$ $\left\{b_{1}, b_{2}, b_{3}, b_{4}, b_{1}, b_{2}, \ldots\right\}$, with $c_{2}<0$ and $c_{4}>0$
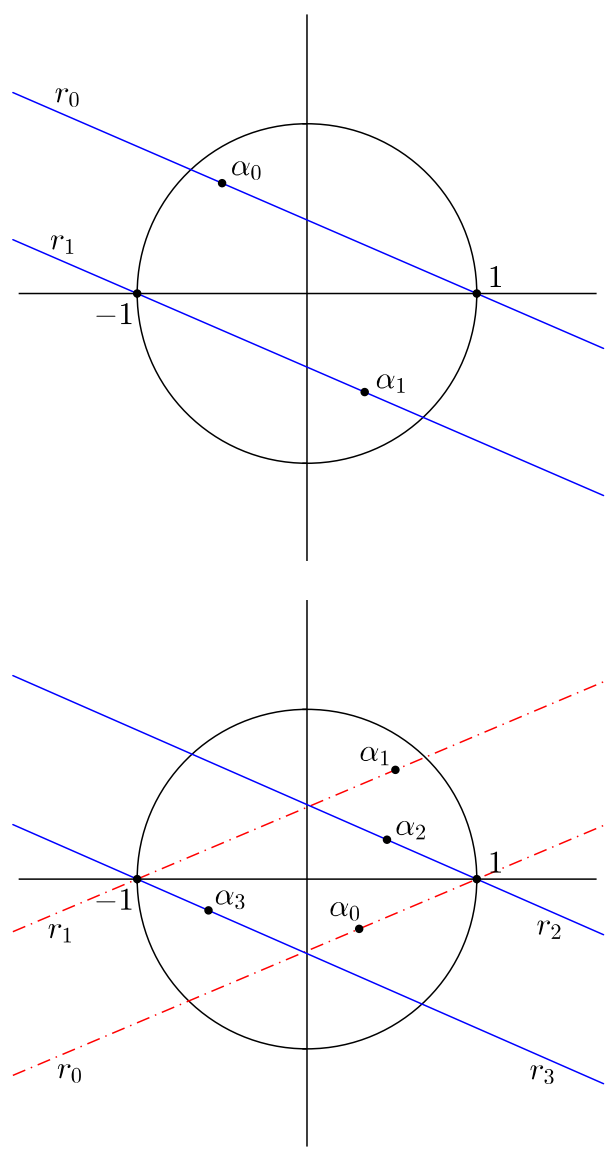

Proof By Theorems 6 and 7, we see that $w$ is a possible pure point of $\mu^{(p)}$ if, and only if, $\varphi_{p}(w)-\varphi_{p}^{*}(w)=0$. Notice that the condition $\varphi_{p}(w)-\varphi_{p}^{*}(w)=0$ is equivalent to $\tau_{p}(w)=1$.

Furthermore, using the periodicity of the sequence $\left\{\alpha_{n}\right\}_{n=0}^{\infty}$ and the recurrence relation (6), we also see that $\tau_{p}(w)=1$ is equivalent to the periodicity of the sequence $\left\{\tau_{n}(w)\right\}_{n=0}^{\infty}$.

The next theorem provides a way to determine all the pure points of the measure $\mu^{(p)}$ and, also, to calculate the mass of each pure point.

Theorem 13 Let $\mu^{(p)}$ be a probability measure on the unit circle with associated $p$-periodic sequence of Verblunsky coefficients $\left\{\alpha_{n}\right\}_{n=0}^{\infty}$. In addition, suppose that $w$ is a point on the unit circle such that $\varphi_{p}(w)-\varphi_{p}^{*}(w)=0$. Then, $w$ is a pure point of $\mu^{(p)}$ if, and only if,

$$
\prod_{j=1}^{p}\left|1-w \tau_{j-1}(w) \alpha_{j-1}\right|^{2}<\prod_{j=1}^{p}\left[1-\left|\alpha_{j-1}\right|^{2}\right] .
$$

Moreover, if $w$ is a pure point of $\mu^{(p)}$, then the mass at this point is given by

$$
\mu^{(p)}(\{w\})=\frac{\gamma}{\gamma+\delta},
$$


where $\delta=\sum_{n=1}^{p} \prod_{j=1}^{n} \frac{\left|1-w \tau_{j-1}(w) \alpha_{j-1}\right|^{2}}{1-\left|\alpha_{j-1}\right|^{2}}$ and $\gamma=1-\prod_{j=1}^{p} \frac{\left|1-w \tau_{j-1}(w) \alpha_{j-1}\right|^{2}}{1-\left|\alpha_{j-1}\right|^{2}}$.

Proof For $j \geq 1$, let $q_{j}=\frac{\left|1-w \tau_{j-1}(w) \alpha_{j-1}\right|^{2}}{1-\left|\alpha_{j-1}\right|^{2}}$.

By Theorem 2, we know that $w$ is a pure point if, and only if, the infinite sum $\lambda(w)=$ $\sum_{n=1}^{\infty} \prod_{j=1}^{n} q_{j}$ is convergent.

By Lemma 4 and the periodicity of $\left\{\alpha_{n}\right\}_{n=0}^{\infty}$, it follows that $q_{j+p}=q_{j}, j \geq 1$. Thus, if $q=\prod_{j=1}^{p} q_{j}$, we can write $\lambda(w)$ as

$$
\lambda(w)=q_{1}\left(\sum_{n=0}^{\infty} q^{n}\right)+q_{1} q_{2}\left(\sum_{n=0}^{\infty} q^{n}\right)+\cdots+q_{1} q_{2} \cdots q_{p}\left(\sum_{n=0}^{\infty} q^{n}\right) .
$$

Observe that $\lambda(w)$ is convergent if, and only if, $|q|<1$. Thus, the first part of the statement follows.

Furthermore, if $|q|<1$ using (33), we have

$$
\lambda(w)=\left(\frac{1}{1-q}\right)\left(\sum_{n=1}^{p} \prod_{j=1}^{n} q_{j}\right)=\frac{\delta}{\gamma} .
$$

Finally, by Theorem 2 and (34), we get

$$
\mu^{(p)}(\{w\})=\frac{1}{1+\lambda(w)}=\frac{\gamma}{\gamma+\delta} .
$$

\section{An example}

In this section, we discuss, using the following example, the results obtained in the previous sections.

Let the real sequences $\left\{c_{n}\right\}_{n=1}^{\infty}$ and $\left\{d_{n}\right\}_{n=1}^{\infty}$ be given by

$$
c_{n}=(-1)^{n} c \text { and } d_{n}=\left(1-m_{n-1}\right) m_{n}, \quad n \geq 1,
$$

where $c \in \mathbb{R}$ and the real sequence $\left\{m_{n}\right\}_{n=0}^{\infty}$ is such that $m_{0}=0$,

$$
m_{2 n-1}=\frac{1-b_{1}}{2} \text { and } m_{2 n}=\frac{1-b_{2}}{2}, \quad n \geq 1,
$$

with $b_{1}, b_{2} \in \mathbb{R},\left|b_{1}\right|<1$ and $\left|b_{2}\right|<1$.

Notice that, if $c \neq 0,\left\{c_{n}\right\}_{n=1}^{\infty}$ has the alternating sign property and that $\left\{d_{n}\right\}_{n=1}^{\infty}$ is a positive chain sequence, with $\left\{m_{n}\right\}_{n=0}^{\infty}$ being its minimal parameter sequence. Moreover, $\left\{c_{n}\right\}_{n=1}^{\infty}$ and $\left\{m_{n}\right\}_{n=1}^{\infty}$ are periodic sequences of period 2 .

By Theorem 1, associated with the pair $\left\{\left\{c_{n}\right\}_{n=1}^{\infty},\left\{d_{n}\right\}_{n=1}^{\infty}\right\}$, there exists a unique probability measure, say $\mu^{(2)}$, on the unit circle. Furthermore, from Corollary 3 it follows that the sequence of Verblunsky coefficients associated with $\mu^{(2)}$ is periodic with period 2 (in Fig. 1, the position of these coefficients for the case $c>0$ is illustrated).

From the definition of $\left\{c_{n}\right\}_{n=1}^{\infty}$, one can also see that

$$
\tau_{2 n}=1 \quad \text { and } \quad \tau_{2 n+1}=\frac{1+i c}{1-i c}, n \geq 0 .
$$


Thus, from (5) we have, for $n \geq 0$,

$$
\begin{aligned}
\alpha_{2 n} & =\frac{b_{1}+i c}{1+i c}=\frac{\left(b_{1}+c^{2}\right)+i c\left(1-b_{1}\right)}{1+c^{2}} \text { and } \\
\alpha_{2 n+1} & =\frac{b_{2}-i c}{1+i c}=\frac{\left(b_{2}-c^{2}\right)-i c\left(1+b_{2}\right)}{1+c^{2}} .
\end{aligned}
$$

In this case, since $p=2$, we have $\Delta(z)=z^{-1} \operatorname{Tr}\left(T_{2}(z)\right)$. By (7) and (8),

$$
T_{2}(z)=\left(1-\left|\alpha_{0}\right|^{2}\right)^{-1 / 2}\left(1-\left|\alpha_{1}\right|^{2}\right)^{-1 / 2}\left(\begin{array}{cc}
z & -\bar{\alpha}_{1} \\
-\alpha_{1} z & 1
\end{array}\right)\left(\begin{array}{cc}
z & -\bar{\alpha}_{0} \\
-\alpha_{0} z & 1
\end{array}\right) .
$$

Hence, computing $\Delta\left(e^{i \theta}\right)$ one can see that, for $\theta \in[0,2 \pi)$,

$$
\Delta\left(e^{i \theta}\right)=2\left\{\frac{1+c^{2}}{\left[\left(1-b_{1}^{2}\right)\left(1-b_{2}^{2}\right)\right]^{1 / 2}} \cos \theta+\frac{b_{1} b_{2}-c^{2}}{\left[\left(1-b_{1}^{2}\right)\left(1-b_{2}^{2}\right)\right]^{1 / 2}}\right\}
$$

and, consequently,

$$
\sqrt{4-\Delta^{2}\left(e^{i \theta}\right)}=2 \sqrt{1-\left[\frac{\left(1+c^{2}\right) \cos \theta+b_{1} b_{2}-c^{2}}{\left(1-b_{1}^{2}\right)^{1 / 2}\left(1-b_{2}^{2}\right)^{1 / 2}}\right]^{2}} .
$$

Furthermore, by considering the normalized orthogonal polynomials $\varphi_{2}(z)=\kappa_{2} \phi_{2}(z)$ one can also verify that

$$
\begin{aligned}
\varphi_{2}(z)= & \frac{1}{\left(1-b_{1}^{2}\right)^{1 / 2}\left(1-b_{2}^{2}\right)^{1 / 2}}\left\{\left(1+c^{2}\right) z^{2}+\left(b_{1} b_{2}-b_{1}-2 c^{2}\right) z\right\} \\
& +\frac{1}{\left(1-b_{1}^{2}\right)^{1 / 2}\left(1-b_{2}^{2}\right)^{1 / 2}}\left\{i c\left(b_{2}+1\right) z+\left[\left(c^{2}-b_{2}\right)-i c\left(b_{2}+1\right)\right]\right\}
\end{aligned}
$$

and, consequently, for $\theta \in[0,2 \pi)$,

$$
\operatorname{Im}\left(e^{-i \theta} \varphi_{2}\left(e^{i \theta}\right)\right)=\frac{\left(1+b_{2}\right)[\sin \theta+c(1-\cos \theta)]}{\left(1-b_{1}^{2}\right)^{1 / 2}\left(1-b_{2}^{2}\right)^{1 / 2}} .
$$

Hence, from Theorem 5, the weight function $w(\theta)$ associated with $\mu^{(2)}$ is such that

$$
w(\theta)=\frac{\sqrt{\left(1-b_{1}^{2}\right)\left(1-b_{2}^{2}\right)-\left[\left(1+c^{2}\right) \cos \theta+b_{1} b_{2}-c^{2}\right]^{2}}}{\left|\left(1+b_{2}\right)[\sin \theta+c(1-\cos \theta)]\right|} .
$$

Now, we need to compute the bands $B_{1}$ and $B_{2}$ for the measure $\mu^{(2)}$. By solving the equation $\Delta\left(e^{i \theta}\right)=2$, we find the solutions

$$
\theta_{1}^{+}=\arccos \left(\frac{\left(1-b_{1}^{2}\right)^{1 / 2}\left(1-b_{2}^{2}\right)^{1 / 2}+c^{2}-b_{1} b_{2}}{1+c^{2}}\right) \text { and } \theta_{2}^{+}=2 \pi-\theta_{1}^{+} .
$$

Likewise, by solving $\Delta\left(e^{i \theta}\right)=-2$, we find

$$
\theta_{1}^{-}=\arccos \left(\frac{c^{2}-\left(1-b_{1}^{2}\right)^{1 / 2}\left(1-b_{2}^{2}\right)^{1 / 2}-b_{1} b_{2}}{1+c^{2}}\right) \text { and } \theta_{2}^{-}=2 \pi-\theta_{1}^{-} \text {. }
$$

Thus, each band $B_{j}$ is determined by the points $z_{j}^{+}=e^{i \theta_{j}^{+}}$and $z_{j}^{-}=e^{i \theta_{j}^{-}}, j \in\{1,2\}$. 
To determine the possible pure points of $\mu^{(2)}$, by Theorems 6 and 7 , we need to solve the equation $\varphi_{2}(z)-\varphi_{2}^{*}(z)=0$, whose solutions are $w_{1}=1$ and $w_{2}=\frac{c^{2}-1}{1+c^{2}}-i \frac{2 c}{1+c^{2}}$.

Now, looking at the bands $B_{j}$ and at the possible pure points $w_{j}$, it is not hard to see that the measure $\mu^{(2)}$ is always supported on $\mathcal{C}_{1} \cup \mathcal{C}_{2}$, in accordance with Theorem 9.

Finally, we give a complete characterization about the singular part of the measure $\mu^{(2)}$ in terms of the parameters $b_{1}, b_{2}$ and $c$.

First, we analyze the point $w_{1}=1$. Notice that $\tau_{n}\left(w_{1}\right)=\tau_{n}$ given by (35) is periodic of period 2, according to Lemma 4. From Theorem 13, one can see that $w_{1}$ is a pure point of $\mu^{(2)}$ if, and only if, $b_{1}+b_{2}>0$. Moreover, if $\delta_{1}=\sum_{n=1}^{2} \prod_{j=1}^{n} \frac{\left|1-\tau_{j-1} \alpha_{j-1}\right|^{2}}{1-\left|\alpha_{j-1}\right|^{2}}$ and $\gamma_{1}=1-\prod_{j=1}^{2} \frac{\left|1-\tau_{j-1} \alpha_{j-1}\right|^{2}}{1-\left|\alpha_{j-1}\right|^{2}}$, again by Theorem 13 we obtain

$$
\mu^{(2)}\left(\left\{w_{1}\right\}\right)=\frac{\gamma_{1}}{\gamma_{1}+\delta_{1}}=\frac{b_{1}+b_{2}}{1+b_{2}} .
$$

Consider now the point $w_{2}=\frac{c^{2}-1}{1+c^{2}}-i \frac{2 c}{1+c^{2}}=-\frac{1+i c}{1-i c}$. From Corollary 2, if $\tilde{\mu}(z)=$ $\mu^{(2)}\left(w_{2} z\right)$, we have $\tilde{c}_{n}=c, n \geq 1$. Moreover,

$$
\tilde{\tau}_{n}=\prod_{k=1}^{n} \frac{1-i \tilde{c}_{k}}{1+i \tilde{c}_{k}}=\left(\frac{1-i c}{1+i c}\right)^{n}, \quad n \geq 1 .
$$

Fig. 3 Case $0<c<1$ and $b_{2}>b_{1}>0$

Fig. 4 Case $0<c<1$ and $0<b_{2} \leq-b_{1}$
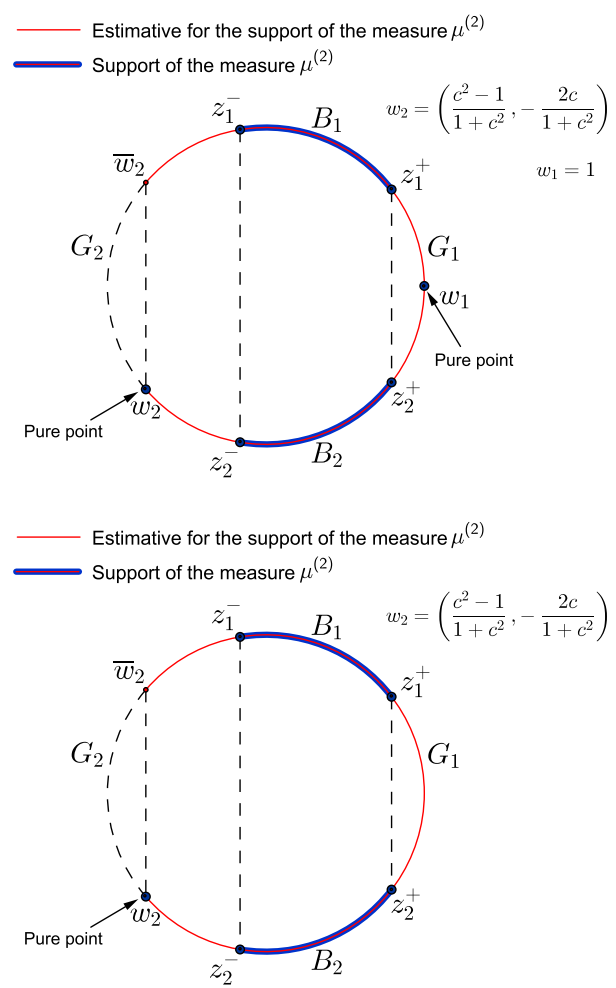
Fig. 5 Case $0<c<1$ and $0<b_{2} \leq b_{1}$

Fig. 6 Case $0<c<1$ and $b_{1}=b_{2}=0$

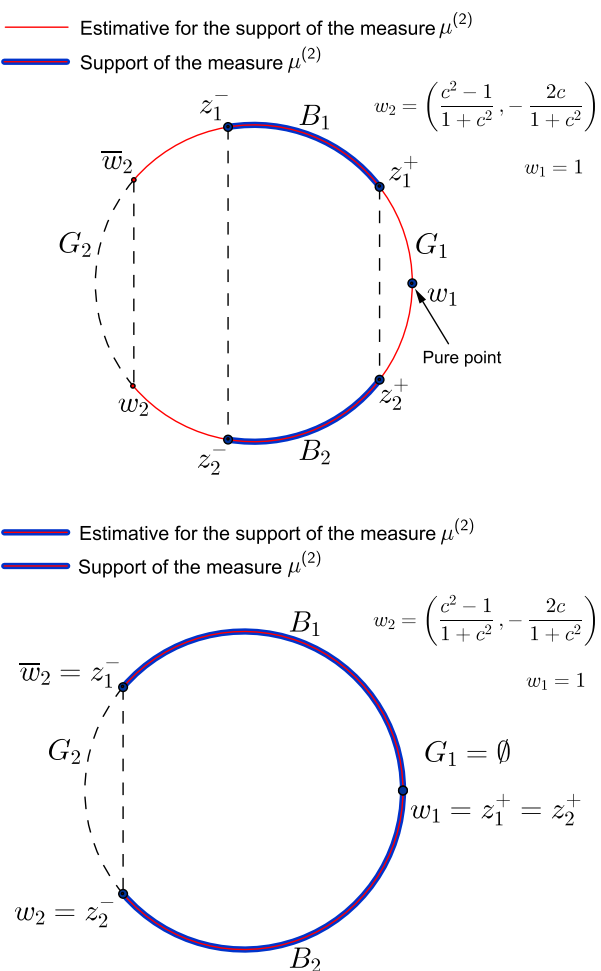

On the other hand, it is known (see, for example, Costa et al. (2013)) that $\tilde{\tau}_{n}=$ $w_{2}^{-n} \tau_{n}\left(w_{2}\right), n \geq 0$. Hence, one can see that $\tau_{n}\left(w_{2}\right)=(-1)^{n}, n \geq 0$. Thus, it follows that $\tau_{n}\left(w_{2}\right)$ is periodic of period 2, according to Lemma 4 .

From Theorem 13, $w_{2}$ is a pure point of $\mu^{(2)}$ if, and only if, $b_{2}-b_{1}>0$. Moreover, if

$$
\delta_{2}=\sum_{n=1}^{2} \prod_{j=1}^{n} \frac{\left|1-w_{2} \tau_{j-1}\left(w_{2}\right) \alpha_{j-1}\right|^{2}}{1-\left|\alpha_{j-1}\right|^{2}} \text { and } \gamma_{2}=1-\prod_{j=1}^{2} \frac{\left|1-w_{2} \tau_{j-1}\left(w_{2}\right) \alpha_{j-1}\right|^{2}}{1-\left|\alpha_{j-1}\right|^{2}}
$$

we obtain

$$
\mu^{(2)}\left(\left\{w_{2}\right\}\right)=\frac{\gamma_{2}}{\gamma_{2}+\delta_{2}}=\frac{b_{2}-b_{1}}{1+b_{2}} .
$$

The Figs. 3, 4, 5 and 6 illustrate a comparison between the estimative for the support of the measure $\mu^{(2)}$ obtained in Theorem 2 (see also Theorem 3) and the true support of the measure $\mu^{(2)}$ in some particular cases. Notice that in the case $b_{1}=b_{2}=0$, the estimative is accurate, i.e., the support of the measure $\mu^{(2)}$ is exactly $\mathcal{C}_{1} \cup \mathcal{C}_{2}$ (see Fig 6).

\section{References}

Bracciali CF, McCabe JH, Peréz TE, Sri Ranga A (2016) A class of orthogonal functions given by a three term recurrence formula. Math Comput 85:1837-1859

Breuer J, Ryckman E, Simon B (2010) Equality of the spectral and dynamical definitions of reflection. Commun Math Phys 295:531-550 
Castillo K, Costa MS, Sri Ranga A, Veronese DO (2014) A Favard type theorem for orthogonal polynomials on the unit circle from a three term recurrence formula. J Approx Theory 184:146-162

Castillo K, Garza L, Marcellán F (2011) Perturbations on the subdiagonals of Toeplitz matrices. Linear Algebra Appl 434:1563-1579

Chihara TS (1978) An introduction to orthogonal polynomials. Gordon and Breach, New York

Costa MS, Felix HM, Sri Ranga A (2013) Orthogonal polynomials on the unit circle and chain sequences. J Approx Theory 173:14-32

Costa MS, Godoy E, Lamblém RL, Sri Ranga A (2011) Basic hypergeometric functions and orthogonal Laurent polynomials. Proc Am Math Soc 140:2075-2089

Dimitrov DK, Sri Ranga A (2013) Zeros of a family of hypergeometric para-orthogonal polynomials on the unit circle. Math Nachr 286:1778-1791

Erdélyi T, Nevai P, Zhang J, Geronimo J (1991) A simple proof of "Favard's theorem" on the unit circle. Atti Semin Mat Fis Univ Modena Reggio Emilia 39:551-556

Geronimus Ya L (1944) On polynomials orthogonal on the unit circle, on trigonometric moment problem, and on allied Carathéodory and Schur functions. Mat Sb 15:99-130

Ismail MEH (2005) Classical and quantum orthogonal polynomials in one variable. In: Encyclopedia of mathematics and its applications, vol 98. Cambridge University Press, Cambridge

Kheifets A, Golinskii L, Peherstorfer F, Yuditskii P (2011) Scattering theory for CMV matrices: uniqueness, Helson-Szegő and strong Szegő theorems. Integral Equ Oper Theory 69:479-508

Peherstorfer F (2011) Positive trigonometric quadrature formulas and quadrature on the unit circle. Math Comput 80:1685-1701

Peherstorfer F, Steinbauer R (1996) Orthogonal polynomials on arcs of the unit circle, I. J Approx Theory $85: 140-184$

Peherstorfer F, Steinbauer R (1996) Orthogonal polynomials on arcs of the unit circle, II. Orthogonal polynomials with periodic reflection coefficients. J Approx Theory 87:60-112

Peherstorfer F, Volberg A, Yuditskii P (2009) CMV matrices with asymptotically constant coefficients. SzegőBlaschke class, scattering theory. J Funct Anal 256:2157-2210

Simanek B (2012) Week convergence of CD kernels: a new approach on the circle and real line. J Approx Theory 164:204-209

Simon B (2005) Orthogonal polynomials on the unit circle. Part 1. Classical theory. In: American Mathematical Society Colloquium Publications, vol 54. American Mathematical Society, Providence, RI

Simon B (2005) Orthogonal polynomials on the unit circle. Part 2. Spectral theory. In: American Mathematical Society Colloquium Publications, vol 54. American Mathematical Society, Providence, RI

Szegő G (1975) Orthogonal polynomials. In: American Mathematical Society Colloquium Publications, vol 23, 4th edn. American Mathematical Society, Providence, RI

Tsujimoto S, Zhedanov A (2009) Elliptic hypergeometric Laurent biorthogonal polynomials with a dense point spectrum on the unit circle. SIGMA Symmetry Integrability Geom Methods Appl 5:30

Wall HS (1948) Analytic theory of continued fractions. D. Van Nostrand Company, New York 